\title{
Differential Evolution based Inter-cluster Data Aggregation for Mobile Sink based Heuristic Algorithm for Clustering Hierarchy Protocol
}

\author{
Ramandeep Singh \\ M.Tech, Department of Electronics and \\ Communication Engineering, \\ Khalsa College of Engineering and Technology, \\ Amritsar
}

\begin{abstract}
Wireless sensor network (WSNs) are promising while important as well as common strategies to furnishing pervasive computing surroundings for several applications. Unbalanced use of power is surely an normal problem in WSNs, described as multi-hop routing as well as many-to-one visitors pattern. This irregular power dissipation might significantly minimize the system lifetime. So in the effort to enhance the network life-time along with minimizing energy dissipation various energy-efficient routing protocols are designed for the actual sensor networks. Hierarchical routing follows the clustering mechanism which is considered to be efficient in terms of energy and scalability. In this paper, a comparison of different parameters used in Differential evolution based inter-cluster data aggregation for mobile sink based heuristic algorithm for clustering hierarchy protocol are considered and the techniques used i.e. HEED, DWEHC, LEACH.
\end{abstract}

\section{General Terms}

Wireless sensor networks, clustering

\section{Keywords}

Differential evolution, Energy Efficiency

\section{INTRODUCTION}

In the modern era, there is a need for a world of fully connected devices. Networking is considered to be the fastest growing field in the area of research. Remote correspondences have brought the colossal unrest as it amplifies the abilities of different sorts of altered systems which incorporates area autonomous data stockpiling, transport, recovery, preparing and to help the clients to move openly starting with one area then onto the next [1]. Recent scientific and technical advancements in the field of networking have enabled us to produce a very small size, Cheap and battery-operated sensor nodes. These types of nodes can sense the earth and collect the information and forward through numerous jumps. Sensor hubs measure totally concealing situation with in the encompassing atmosphere which can be utilized to depict the qualities of the marvels happening at the area where the sensor hubs are appropriated efficiently. An extensive number of sensor hubs are than set discretionarily over a topographical locale and organized through remote connections so as to arrange a remote sensor arrange. Every sensor hub is than ready to collaborate with each other and base stations are than used to incorporate and telecast the information further. Wireless networking is a significant structure which is used to aid the specifications of armed

\author{
Parmbir Singh \\ M.Tech, Department of Electronics and \\ Communication Engineering, \\ Khalsa College of Engineering and Technology, \\ Amritsar
}

forces and industrial services. Many types of restraints including storage capacity or energy supplies are considered to be important issues [2]. There are many provisions which can keep back the strength of WSNs to assist different applications including sensor devices and limited battery power. To eradicate the difficulty or to balance the WSN load, energy consumption should be reduced and life time of nodes should be maximized.

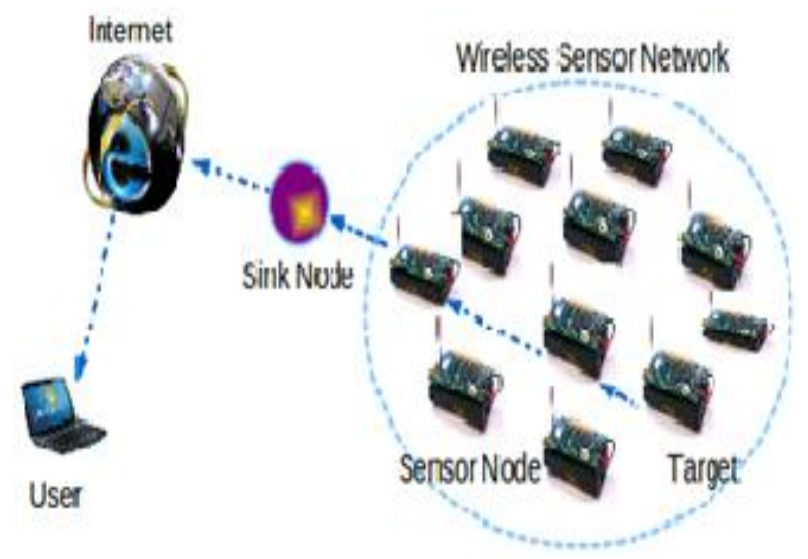

Fig 1: Wireless Sensor Network

\section{HISTORICAL BACKGROUND}

It discussed different energy efficient routing protocols that are based on clustering mechanism. It divides the area into several zones according to the distance and uses the clustering method among these zones. The basic idea of these conventions was that cluster head within the group gets the information from remaining hubs and such information was given to base station [10]. It has talked about some real applications as planning minimal effort secured Intelligent Buildings, In-Home Health consideration and Agriculture. Along with this the classification and challenges of the Next Generation Wsn has also been discussed in this paper [11]. It resented road and rail network fitness monitoring application that uses sensor networks to gather the data about the structural well being along with actions of the infrastructure when a train travels along it and transfers the readings to a base station. The base station then uses the following train(s) as a information mule to transfer the data. The assessment completed demonstrates that the versatile information exchange is really possible and that the outcomes acquired are agreeable, both regarding dependability and power utilization [12] It has analyzed a few procedures that adjust the vitality utilization of hubs and guarantee maximum lifetime by adjusting the load as similarly as could reasonably be 
expected. Furthermore comparison has been done between no. of load balancing strategies that are used in wireless sensor networks[5]. In proposed a Tree based routing protocol algorithm that helps to construct steering hierarchy utilizing a procedure in which base Station designates a source hub and shows its determination to other alarming hubs. In this each center picks its guardian by considering just itself and its neighbors' information, thusly making [17] Tree based routing protocol a dynamic tradition. Result shows that Tree based routing protocol has shown excellent results as compared to other protocols and helps in increasing the lifetime of whole sensor network [4]. It compressed the assaults and orders in remote sensor systems. An effort has been made to investigate the security instrument broadly used to handle those assaults. The master plan of this paper had been to examine the security related problems, the difficulties and to propose a few arrangements with a specific end goal to secure the WSN against the security dangers [7].In this paper, we discussed that in wireless body area networks, all the alarming nodes operate very close to each other either on or inside the body of a person [16]. They can check bp, blockage, clotting, heart attacks etc. These nodes have very limited power and batteries can neither be changed nor charged. So, energy consumption model has been discussed which helps in increasing life of an alarming node [3]. Some sort of 3-level heterogeneous network model to get WSNs to further improve the particular network lifelong is definitely carried out, that is seen a particular parameter. Based on value of the particular design parameter, it may possibly identify 1-level, 2-level, as well as 3-level heterogeneity [15]. Heterogeneous system design can also help to choose cluster heads along with particular cluster members by utilizing calculated election possibility and limit function. The particular network life-time by simply using DEEC method due to this design is also implemented [8]. It demonstrated a novel bio-inspired routing protocol, named CB-RACO that combines the Ant Colony Optimization (ACO) meta-heuristic with the computationally cheap and distributed community detection technique Label Propagation (LP) [14]. CB-RACO creates communities in the WSNs and meets the balance of energy consumption by routing data inside-communities through swarm intelligence. As a consequence, CB-RACO demands low memory and overhead in construction and maintenance of routing paths. Additionally, CB-RACO achieves high data delivery reliability through a data retransmission strategy based on acknowledgments between communities [6]. It implemented a new novel for Clustering Hierarchy $(\mathrm{HACH})$, which usually sequentially carries out collection of non-active nodes as well as cluster head nodes at each round. Non-active node collection uses a new stochastic sleeping arrangement process to find out selecting a nodes that could be place into sleeping method without having negatively heaving an effect on network coverage [13]. As well, a clustering algorithm criteria utilizes a story heuristic crossover user to mix a pair of several methods to accomplish a much better answer this improves the circulation connected with cluster head nodes and also coordinates energy utilization throughout WSNs [9].

Table No. 1 Recent advancement in inter-cluster data aggregation for mobile sink based heuristic protocols

\begin{tabular}{|c|c|c|}
\hline Title of the paper & Advantages & Disadvantages \\
\hline $\begin{array}{l}\text { Energy efficient transmission approach for } \\
\text { WBAN based on threshold distance. [3] }\end{array}$ & $\begin{array}{l}59.77 \% \text { or higher energy saving can } \\
\text { be possible with the optimized } \\
\text { scheme, compared to baseline scheme, } \\
\text { overall energy utilization is usually } \\
\text { [11]. }\end{array}$ & $\begin{array}{l}\text { It will require higher range of the } \\
\text { transmitting power within the network } \\
\text { while transmission [12]. }\end{array}$ \\
\hline $\begin{array}{l}\text { VGDRA: A Virtual Grid based Dynamic } \\
\text { Routes Adjustment Scheme for Mobile Sink } \\
\text { based Wireless Sensor Networks [4] }\end{array}$ & $\begin{array}{l}\text { Good strategy to balance the nodes } \\
\text { energy dissipation, efficient data } \\
\text { delivery, Show decreased channels } \\
\text { reconstruction cost and also increased } \\
\text { network life-time, decreasing the } \\
\text { overall communication cost [10]. }\end{array}$ & $\begin{array}{l}\text { The information distribution towards the } \\
\text { mobile phone sink is really a difficult job } \\
\text { for the source for little sensor nodes } \\
\text { because of the dynamic network topology } \\
\text { induced while using the sink mobility. }\end{array}$ \\
\hline $\begin{array}{l}\text { Comparative study of load balancing } \\
\text { techniques for optimization of network } \\
\text { lifetime in wireless sensor networks.[5] }\end{array}$ & $\begin{array}{l}\text { Select a best path for information } \\
\text { transmitting and take a smaller amount } \\
\text { of energy [9]. }\end{array}$ & Applies additional load to a unique node. \\
\hline $\begin{array}{l}\text { Enhancing the reliability on data delivery } \\
\text { and energy efficiency by combining swarm } \\
\text { intelligence and community detection in } \\
\text { large-scale WSNs.[6] }\end{array}$ & $\begin{array}{l}\text { Operate the network autonomously } \\
\text { with very low network overload and } \\
\text { memory cost [8]. }\end{array}$ & Designing cost is high \\
\hline $\begin{array}{l}\text { Wireless sensor networks: security issues, } \\
\text { challenges and solutions. [7] }\end{array}$ & $\begin{array}{l}\text { Security in Wireless Sensor Network } \\
\text { is better than traditional different } \\
\text { method [7]. }\end{array}$ & $\begin{array}{l}\text { It can't able to use security methods } \\
\text { similar to wired networks. }\end{array}$ \\
\hline $\begin{array}{l}\text { Energy efficient heterogeneous DEEC } \\
\text { protocol for enhancing lifetime in WSNs. [8] }\end{array}$ & $\begin{array}{l}\text { Improving the network energy in } \\
\text { addition to utilizing the network } \\
\text { energy successfully raises the network } \\
\text { lifetime. }\end{array}$ & $\begin{array}{l}\text { It needs higher range from the } \\
\text { transmitting energy from the network } \\
\text { while transmission [6]. }\end{array}$ \\
\hline
\end{tabular}




\begin{tabular}{|c|c|c|}
\hline $\begin{array}{l}\text { HACH: Heuristic Algorithm for Clustering } \\
\text { Hierarchy protocol in wireless sensor } \\
\text { networks. [9] }\end{array}$ & $\begin{array}{l}\text { Performs collection of non-active } \\
\text { nodes and cluster head nodes at each } \\
\text { and each round and balances energy } \\
\text { consumption. }\end{array}$ & $\begin{array}{l}\text { Wireless sensors used in agriculture, } \\
\text { Medical as well as developing high cost } \\
\text { secured intelligent building for } \\
\text { developing countries is a difficult task. }\end{array}$ \\
\hline $\begin{array}{l}\text { Energy optimization at the MAC layer for a } \\
\text { forest fire monitoring wireless sensor network. } \\
{[10]}\end{array}$ & $\begin{array}{l}\text { Save power, raise data transfer due to } \\
\text { increase the bandwidth and keeping } \\
\text { power utilization is very low. }\end{array}$ & $\begin{array}{l}\text { The information distribution towards the } \\
\text { mobile sink will be a difficult job for the } \\
\text { particular source limited sensor nodes } \\
\text { because of the dynamic network topology } \\
\text { induced with the sink mobility. }\end{array}$ \\
\hline $\begin{array}{l}\text { Challenges of next-generation wireless } \\
\text { sensor networks and its impact on society. } \\
{[11]}\end{array}$ & Reduces transmission costs & Designing cost is high \\
\hline $\begin{array}{l}\text { The announcement layer: Beacon } \\
\text { coordination for the sensor net stack. [12] }\end{array}$ & Less cost & $\begin{array}{l}\text { Just one most effective route sets } \\
\text { additional load to some unique node. }\end{array}$ \\
\hline
\end{tabular}

\section{CLUSTERING}

When several sensors cooperatively observe huge real environment, that they kind of wireless sensor network. Long term network life-time, scalability, load handling are very important requirement of several sensor network applications. Clustering sensor nodes is an efficient way of reaching these kinds of goals [22].

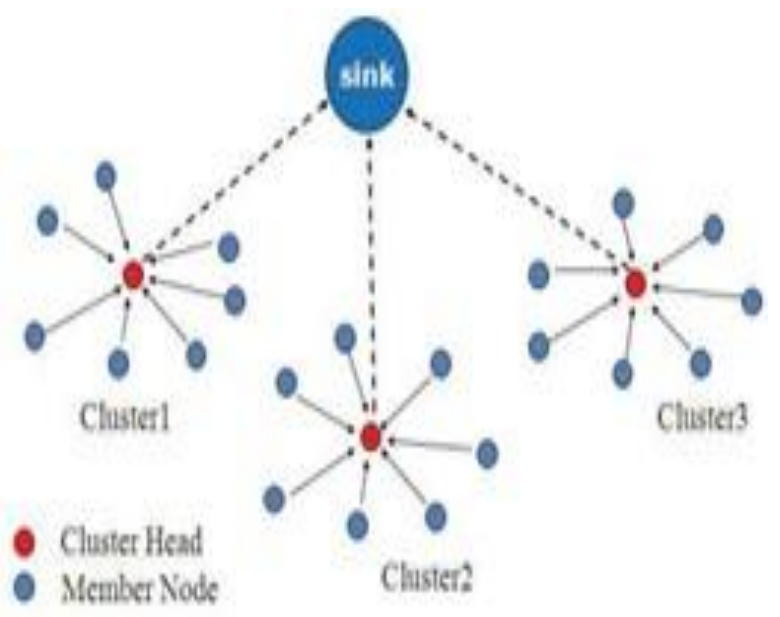

Fig 2: Cluster Head

- $\quad$ Clustering technique is one of the well-liked systems through which nodes pick a Cluster Head $(\mathrm{CH})$ with regard to communication [21].

- $\quad$ All nodes distributed their own information to you to $\mathrm{CH}$ where, that collected info as well as post to your Base Station (BS) [20].

- Only several nodes are widely-used to distribute at large long distance therefore, a lesser amount of energy will be consumed.

- The primary concept of clustering is usually to reduce the actual network traffic coming from node to base station. Cluster head is really a node that is reliable for manage cluster, collect information coming from nodes from the cluster and also communicate with sink.

Clustering can be performed with 2 types of networks: Homogeneous networks, Heterogeneous networks.
The networks which all sensor networks have same amount of energy are called Homogeneous Network. In Heterogeneous clustering the network which some of the sensor network have extra power as compared to other nodes in the network are called Heterogeneous Network [19].

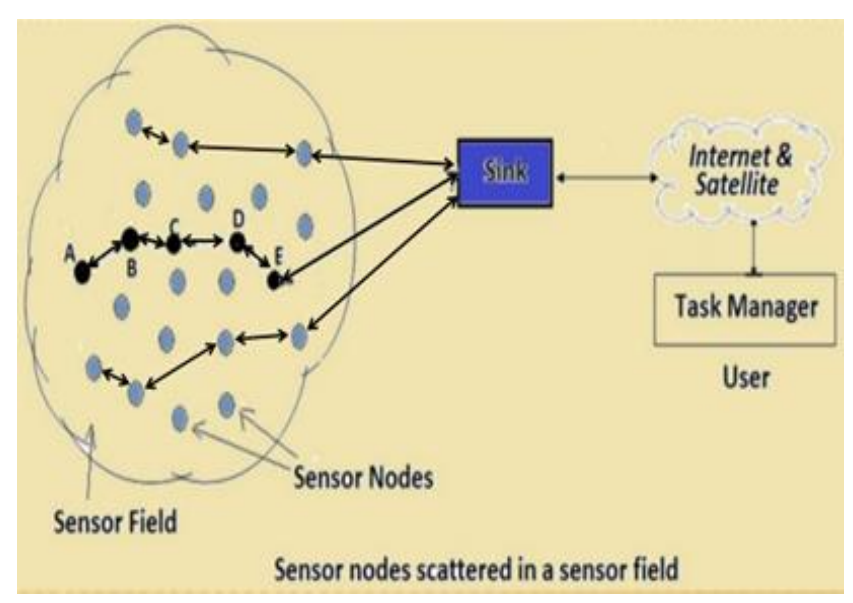

Fig 3: Homogeneous wireless sensor networks

The characteristics of Heterogeneous WSNs algorithms are better than the Homogeneous WSNs in term of the first node dies and the number of packets sent to the base station. Heterogeneous WSNs cluster-based protocols have the better ability to manage the clusters and their member nodes and can better balance the energy consumption of the nodes in the whole network than Homogeneous WSNs energy [18]. 


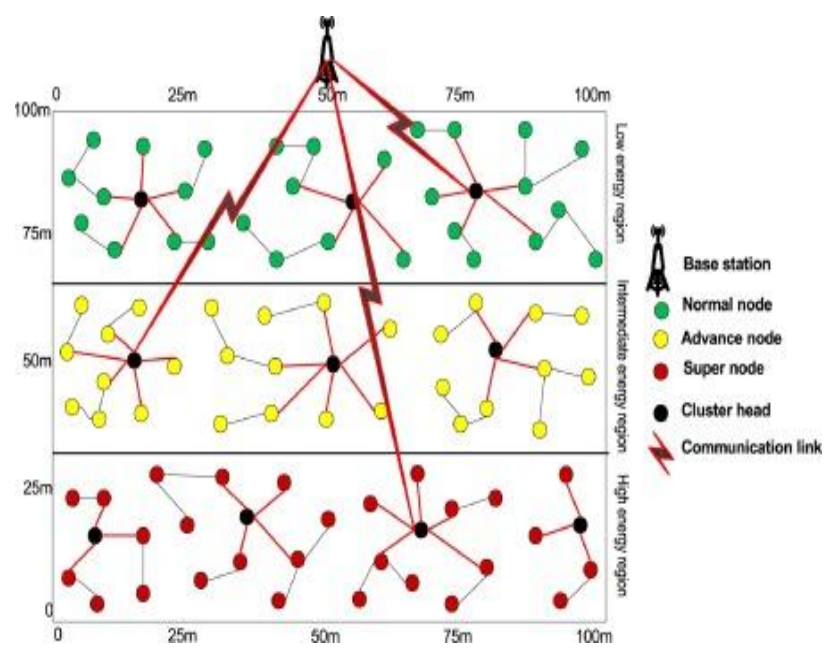

Fig 4: Heterogeneous

\section{ENERGY EFFICIENT ROUTING PROTOCOLS}

\subsection{Low Energy Adaptive Clustering} Hierarchy

LEACH is a well known energy efficient versatile clustering criteria in which types node clusters depending on the good acquired transmission strength.

With LEACH the actual nodes sort area clusters and among this nodes performing as being an area sink or maybe cluster head.

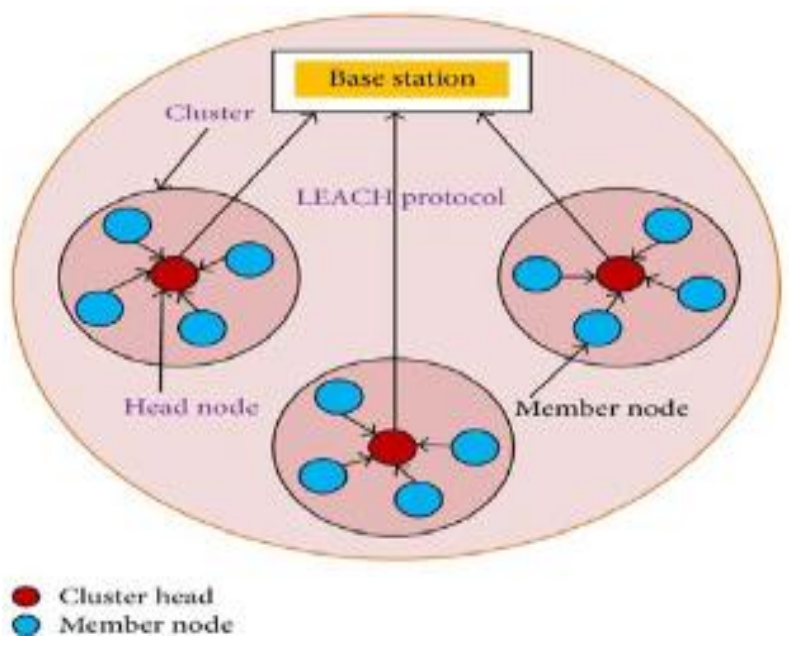

Fig 6: Low Energy Adaptive Clustering Hierarchy

When precisely the same node would certain stay because cluster head all over the running on the network, it might pass away rapidly due to the extensive load with the engaging sensor inside the cluster.

For this reason the particular revolving with the cluster head in every around is definitely essential to disperse the load uniformly. Additional energy dissipation may be decreased by means of aggregating the information out of various sensor / probe nodes at the cluster head.

\subsection{Hybrid Energy-Efficient Distributed Clustering}

HEED is a multi-hop WSN clustering protocol that will be actually provides a good energy effective clustering routing by utilizing from time to time a exactly stress connected with energy [5]. It's totally different from LEACH during the practices with $\mathrm{CH}$ commitment, HEED will not choose nodes because $\mathrm{CHs}$ randomly [4].

The best way of cluster create is completed dependant on hybrid collection using a number of parameters. Among the variables relies in across the node's staying around energy, and also other parameter could possibly be the intra-cluster communicating expenditure. In HEED, chosen $\mathrm{CHs}$ include huge standard remaining energy when compared with MNs.

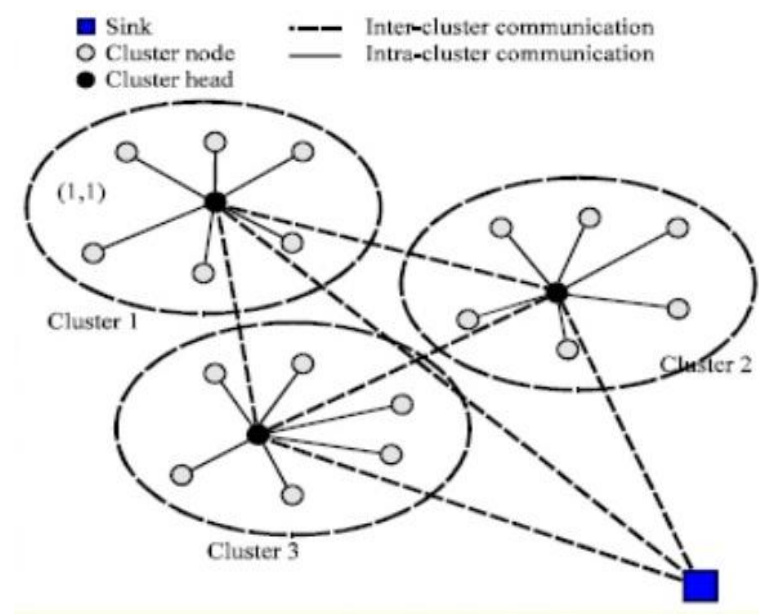

Fig 5: Hybrid Energy-Efficient Distributed Clustering

\subsection{Distributed Weight-based Energy- efficient Hierarchical Clustering protocol}

DWEHC is a dispersed clustering formula for instance HEED. The true secret basis for DWEHC could be to improve HEED simply because develop well balanced cluster measurements as well as improve a new intra-cluster topology through the use of position understanding a new nodes. The two similarly DWEHC as well as HEED focus on several commonalities and also no presumptions in terms of multilevel sizes as well as focus, and employing less than consideration left over energy inside the strategy relating to $\mathrm{CH}$ selection. Almost all nodes uses DWEHC automatically combined with the algorithm indicates immediately after various iterations which can be used in your dispersed manner.

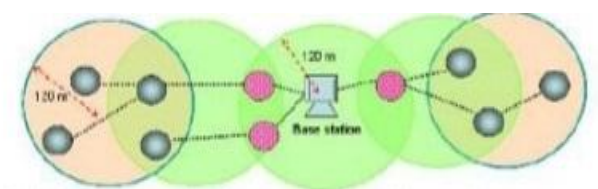

(a) Scenaris 1 the baes station and all seneor nodes have the same trantmisoion range $(120 \mathrm{~m})$

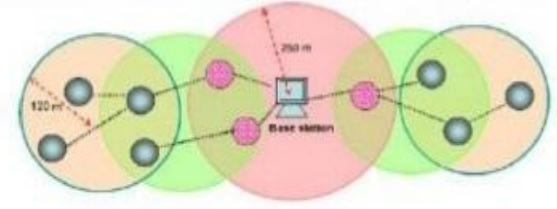

(b) Scenario2: only the transmission range of base station is extended $(250 \mathrm{~m})$

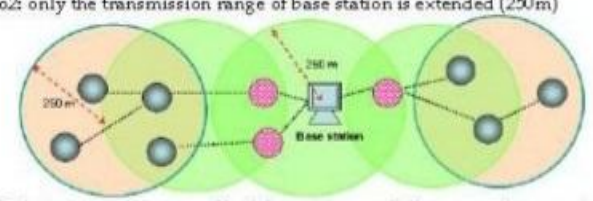

(c) Scenario3: the transmission mange of both base station and all sensor nodes are extended $(250 \mathrm{~m})$ Fig. 14. WSN w with transmiesion mange control

Fig 7: Layout of DWEHC 


\section{COMPARISON OF DIFFERENT SCHEMES}

Table 5 shows that the HACH has better in lifetime, stable period, throughput, packet delivery ratio. Also, HACH has fewer Ends to end delay

\begin{tabular}{|l|l|l|l|}
\hline & HEED & DWEHC & HACH \\
\hline Lifetime & Average & Good & Good \\
\hline Stable period & Good & Average & Good \\
\hline rroughput & Average & Good & Good \\
\hline $\begin{array}{l}\text { Packet delivery } \\
\text { ratio }\end{array}$ & Good & Average & Good \\
\hline End to end delay & Less & Average & Less \\
\hline
\end{tabular}

\section{CONCLUSION}

Wireless network composed of spatially spread autonomous system working with detectors to evaluate physical as well as the environmental conditions. The WSN system contains a path which offers wireless connection back to the community along with distributed nodes. This specific paper shows about the comparison of various techniques based on routing protocols in WSN. This review has reveals that the majority of the established methods neglected the effects of the mobile sink in energy efficient routing protocols, the issue regarding lossless data compression continues to be neglected from the majority of analysts and no optimization method is regarded with the effective path selection in $\mathrm{HACH}$. Therefore in near future we will purpose technique based on energy efficient protocols to overcome the restrictions regarding the previous energyefficient methods utilizing the compressive sensing as well as evolutionary optimization dependent tree structure. Several analytics will likely be utilized to measure the enhancement in offered method above the actual established energy-efficient protocols.

\section{ACKNOWLEDGMENTS}

I am Thankful to my guide Assistant Professor Parambir Singh for his guidance and encouragement for the paper work.

\section{REFERENCES}

[1] Suma.N, Dr. T. Purusothaman. "Enhanced Logical Tree based Routing for Energy Efficient Wireless Sensor Networks", International Journal of Advanced Information Science and Technology (IJAIST), no.8, (2012) :15-19.

[2] Goyal, Deepak, and Malay RanjanTripathy. "Routing protocols in wireless sensor networks: a survey." In 2012 Second International Conference on Advanced Computing \& Communication Technologies, pp. 474-480. IEEE, 2012.

[3] Yi, Chenfu, Lili Wang, and Ye Li. "Energy efficient transmission approach for WBAN based on threshold distance." IEEE Sensors Journal 15, no. 9 (2015): 5133 5141.

[4] Khan, Abdul Waheed, Abdul Hanan Abdullah, Mohammad AbdurRazzaque, and JavedIqbalBangash. "VGDRA: a virtual grid-based dynamic routes adjustment scheme for mobile sink-based wireless sensor networks." IEEE sensors Journal 15, no. 1 (2015): 526-534.
[5] Saraswat, Lalit Kumar, and Sachin Kumar. "Comparative study of load balancing techniques for optimization of network lifetime in wireless sensor networks." IJCER 2, no. 2 (2013): 189-193.

[6] Valerio Rosset, Matheus A. Paulo, Juliana G. Cespedes, Maria C.V. Nascimento, "Enhancing the reliability on data delivery and energy efficiency by combining swarm intelligence and community detection in large-scale WSNs," Expert Systems with Applications, Volume 78, 15 July 2017, Pages 89-102.

[7] Kumar, Vikash, Anshu Jain, and P. N. Barwal. "Wireless sensor networks: security issues, challenges and solutions." International Journal of Information and Computation Technology 4, no. 8 (2014): 859-868.

[8] Samayveer Singh, Aruna Malik, Rajeev Kumar, "Energy efficient heterogeneous DEEC protocol for enhancing lifetime in WSNs," Engineering Science and Technology, an International Journal, Volume 20, Issue 1, February 2017, Pages 345-353.

[9] MuyiwaOlakanmiOladimeji, Mikdam Turkey, Sandra Dudley, "HACH: Heuristic Algorithm for Clustering Hierarchy protocol in wireless sensor networks," Applied Soft Computing, Volume 55, 2017, Pages 452-461.

[10] Dilbag Singh and Vijay Kumar. "Modified gain intervention filter based dehazing technique." Journal of Modern Optics 64, no. 20 (2017): 2165-2178.

[11] Dilbag Singh and Vijay Kumar. "Dehazing of remote sensing images using improved restoration model based dark channel prior." The Imaging Science Journal 65, no. 5 (2017): 282-292

[12] Dilbag Singh and Vijay Kumar. "Comprehensive survey on haze removal techniques." Multimedia Tools and Applications (2017): 1-26.

[13] Dilbag Singh and Vijay Kumar. "Dehazing of remote sensing images using fourth-order partial differential equations based trilateral filter." IET Computer Vision (2017).

[14] Dilbag Singh and Vijay Kumar. "Single image haze removal using integrated dark and bright channel prior." Modern Physics Letters B 32, no. 04 (2018): 1850051.

[15] Dilbag Singh and Vijay Kumar. "Defogging of road images using gain coefficient-based trilateral filter." Journal of Electronic Imaging 27, no. 1 (2018): 013004.

[16] Dilbag Singh Deepak Garg, and Husanbir Singh Pannu. "Efficient landsat image fusion using fuzzy and stationary discrete wavelet transform." The Imaging Science Journal 65 , no. 2 (2017): 108-114.

[17] Sengaliappan, M., and A. Marimuthu. "Improved general self-organized tree-basef routing protocol for wireless sensor network." Journal of Theoretical and Applied Information Technology 68, no. 1 (2014).

[18] Mankar, Gunjan, and Sonali T. Bodkhe. "Traffic aware energy efficient routing protocol." In Electronics Computer Technology (ICECT), 2011 3rd International Conference on, vol. 6, pp. 316-320. IEEE, 2011.

[19] Yogesh Kumar, Rajiv Munjal and Krishan Kumar. "Wireless Sensor Networks and Security Challenges", National Workshop-Cum-Conference on Recent Trends in Mathematics and Computing (RTMC) Proceedings 
published in International Journal of Computer Applications, no.9 (2011):17-21.

[20] Zaid Abdul KaeemNaji, AchalaDeshmukh. "Performance Evaluation of Energy Efficient and Scalable BeeSensor Routing Protocol of Wireless Sensor Networks." IJARCSSE International Journal, (2015): 9-18.

[21] Soleman, Hosam, and Ali Payandeh. "Self-protection mechanism for wireless sensor networks." International
Journal of Network Security \& Its Applications 6, no. 3 (2014): 85-97.

[22] Mundada, Monica R., SavanKiran, ShivanandKhobanna, Raja NahushaVarsha, and Seira Ann George. "A Study on Energy Efficient Routing Protocols in Wireless Sensor Networks." International Journal of Distributed and Parallel Systems (IJDPS) Vol 3 (2012): 311-330. 